\title{
Behavioral assessment of visual deficits in the taiep mutant
}

This article was published in the following Dove Press journal:

Degenerative Neurological and Neuromuscular Disease

9 May 2013

Number of times this article has been viewed

\author{
CM Bloom' \\ RJ Post ${ }^{1,2}$ \\ AM Anch ${ }^{3}$ \\ DG Davenport ${ }^{3}$
}

'Department of Psychology, 2Department of Biology, Providence College, Providence, RI, USA; ${ }^{3}$ Department of Psychology, Saint Louis University, St Louis, MO, USA

Correspondence: Christopher M Bloom Department of Psychology, Providence College, I Cunningham Sq, Providence, RI 029I8, USA

Email cbloom@providence.edu
Abstract: Taiep (tremor, ataxia, immobility, epilepsy, paralysis) mutants show a significant increase in myelin thickness from 10 to 30 days of age but then demonstrate a decrease in myelin thickness from 1 to 6 months. The severity of the demyelination in the optic nerve suggests that visual deficits may exist in the taiep mutants. Animals were trained on a discrimination task, in which responses to a light stimulus (the $\mathrm{S}^{\mathrm{D}}$ period) were reinforced on a fixed ratio (FR)-1 schedule, and responses in the absence of the light stimulus (the $\mathrm{S}^{\Delta}$ period) were not reinforced. Following training, the light intensity presented during the $\mathrm{S}^{\mathrm{D}}$ period was gradually reduced between sessions until -6.0 candela $/ \mathrm{m}^{2}$ was reached. Both groups of animals - taiep mutants and control Sprague Dawley rats - successfully recognized and responded in the presence of the stimulus near perfectly by the final day of training, suggesting that taiep mutants demonstrated normal learning, at least under this paradigm. Despite the severe demyelination of the taiep optic nerve, no visual deficits were detected as both groups of animals performed similarly as the light intensity decreased. Though the myelin loss of the optic nerve may have negatively affected signal transduction, this did not result in an increase in visual threshold.

Keywords: psychophysics, taiep, myelin, discrimination learning, neurodegenerative disease

\section{Introduction}

It has been demonstrated that demyelination of the central nervous system (CNS) produces a slowing and eventual blockage of conduction in the previously myelinated fibers. ${ }^{1,2}$ Saltatory conduction, the process by which myelin increases nerve conductance, is dependent on a number of factors that would be affected by myelin deterioration. A decrease in myelin thickness produces an increase in its capacitance as a result of a decrease in transverse resistance. An increase in current leakage through the remaining myelin results, which delays the excitation at the axonal nodes and, in turn, the rate of action potential propagation. ${ }^{1,2}$ Demyelination results in numerous morphological and behavioral abnormalities in human diseases of myelin, such as multiple sclerosis (MS) and phenylketonuria.

Animal models of myelin diseases have been used successfully for investigation of the anatomical and physiological effects of diseases of myelin. ${ }^{3}$ Unfortunately, the short life span of many of the animal models of myelin disease has made intensive study of the behavioral effects of nervous system demyelination difficult; however, the taiep (tremor, ataxia, immobility, epilepsy, paralysis) rat is unique amongst the myelin mutants, living nearly to full term. This relatively long life span has made it a useful model for investigating behavioral manifestations of myelin disease. ${ }^{4}$ 
An autosomal recessive disorder, the taiep mutation is characterized by a progressive accumulation of microtubules near the smooth endoplasmic reticulum of the oligodendrocytes. ${ }^{5}$ This cytoskeletal abnormality appears to coincide with astrocytosis in the brain and interferes with the intracellular mechanisms necessary to maintain normal CNS myelin. ${ }^{6}$ One of these intracellular mechanisms is likely dopamine activity, as one study has demonstrated that increased binding to D1-like receptors could cause some of the behavioral symptoms seen in the taiep mutant. ${ }^{7}$ Taiep rats demonstrate near-normal levels of myelin at birth but, as the cytoskeletal disorder worsens, a progressive demyelination takes place throughout the CNS. ${ }^{8}$ A number of behavioral effects of the taiep disease have been established, demonstrating a wide range of motor deficits, including both gait and splay abnormalities. ${ }^{9}$

Sensory effects of myelin disease have been demonstrated in human diseases such as MS. Although there is biological evidence to suspect similar dysfunction in the taiep mutant, ${ }^{10}$ no attempts to quantify sensory deficits have been undertaken to date. ${ }^{11}$ Research indicates that the optic nerve of the taiep rat is affected by the developmental demyelination. ${ }^{8}$ In control optic nerves, mean myelin thickness increased significantly with age beginning from 10 days postnatally to 6 months of age. The taiep condition, however, showed normal myelination in the optic nerve only for the first 20 days, after which myelin thickness began deteriorating and continued to do so until 12 months of age. ${ }^{8}$ The importance of the optic nerve in vision is well established, making the visual modality a good candidate for investigation of taiep visual deficits.

While electrophysiology is often used to obtain estimates of visual sensitivity in animals, results have been surprisingly inconsistent. ${ }^{12}$ Behavioral techniques have provided researchers with a second approach to assessing visual deficits in animals. ${ }^{13}$ Many techniques, such as the visual water box method, require that animals recognize a visual cue to choose the proper pathway in a maze-like procedure. ${ }^{14}$ Visual deficits are quantified as a function of the latency to reach the correct maze goal after having recognized the visual cue. Though an effective technique, methods like these are problematic in animals in which the visual deficit is accompanied by a motor deficit. Increased latency to the maze goal is equally likely to be due to the motor deficits as to any visual dysfunction. An alternative technique, centered on operant stimulus-discrimination training, assesses visual function by quantification of behavioral changes in the presence of a light stimulus of varying intensities. Earlier research using similar techniques has demonstrated visual thresholds that agree reasonably well with those derived via visual evoked potentials (VEPs). ${ }^{15}$ This method requires only a single lever-press, a behavior that is easily performed, even by animals with neuromuscular dysfunction.

Because demyelination is known to produce a slowing and eventual blockage of signal transduction in the nervous system, and the importance of the optic nerve in vision is established, demyelination of the optic nerve in the taiep mutant likely results in a lower level of visual discrimination when compared to control rats. In the present study, we trained taiep and control rats on a simple discrimination procedure in which the operant behavior is reinforced only in the presence of a light stimulus. We gradually reduced the intensity of this light signal and compared the frequency of successful discrimination between the two groups. Animals with a visual deficit would be expected to fail to discriminate the light stimulus at a higher magnitude. It is proposed that this technique allows for an assessment of visual deficits that is less susceptible to interference from the motor deficits of taiep.

\section{Methods \\ Subjects}

Fourteen male rats - seven normal Sprague Dawley and seven taiep mutant albino rats - were used in this investigation. Taiep autosomal recessive mutant strain was obtained from the University of Wisconsin-Madison, Madison, WI, USA. The animals were self-mated in our laboratory, housed in pairs, and kept on a 12-hour lightdark cycle.

At 35 days of age, all animals were placed on a food deprivation schedule of $90 \%$ of their ad libitum weight. Initial training on the operant paradigm began at 40 days and continued until 70 days, when testing procedures started. Seventy days was determined to be an appropriate start date because the taiep optic nerve has significantly less myelin than in normal rats by this age. ${ }^{8}$

\section{Apparatus Operant chamber}

Training took place in a standard operant chamber with the light source $11.5 \mathrm{~cm}$ from the floor. The operandum was $6 \mathrm{~cm}$ above the grid floor and the food dispenser was $8.5 \mathrm{~cm}$ from each of the operant chamber's inner walls. 


\section{Photometer}

The intensity of the light stimulus was measured via a photometer (Tektronix, Inc, Beaverton, OR, USA) in footlamberts and later converted to candela $(\mathrm{cd}) / \mathrm{m}^{2}$.

\section{Procedure}

Following food deprivation, animals were trained to press a response lever to receive food reinforcement ( $45 \mathrm{mg}$ pellet). Initial training occurred in the presence of a light stimulus of $-1.0 \log \mathrm{cd} / \mathrm{m}^{2}$ - well above the rodent visual threshold and thus easily detected by both groups of animals.

Following successful lever training, animals were trained on a discrimination task. Daily training periods consisted of 50 signal-light-present $\left(\mathrm{S}^{\mathrm{D}}\right)$ and a varied number of signal-light-not-present $\left(\mathrm{S}^{\Delta}\right)$ trials presented randomly on a variable time (VT) 30-second schedule. $\mathrm{S}^{\mathrm{D}}$ trials consisted of the presentation of a 20 -second light stimulus that signaled and was accompanied by reinforcement in the form of a fixed ratio (FR)-1 schedule delivering a $45 \mathrm{mg}$ food pellet. Each trial ended immediately following reinforcement, at which point the stimulus light was removed. $\mathrm{S}^{\Delta}$ trials (20 seconds) occurred without the presentation of the light stimulus, and responses that occurred in the $\mathrm{S}^{\Delta}$ period were not reinforced. To prevent any accidental contiguity between an $\mathrm{S}^{\Delta}$ response and the onset of the following $\mathrm{S}^{\mathrm{D}}$ period, responses during the last 5 seconds of the $\mathrm{S}^{\Delta}$ period delayed onset of the following trial by an average of 5 seconds (VT-5). A day's session concluded following the 50th $\mathrm{S}^{\mathrm{D}}$ presentation, typically resulting in a total duration of 90 minutes. Discrimination training continued in this way for 8 days, when the animal's behavior reached asymptote.

Training was then adapted to allow for a behavioral measure of visual thresholds. Using the same procedure as in training, the light stimulus presented during the $\mathrm{S}^{\mathrm{D}}$ period was gradually reduced on each subsequent training session in $0.5 \mathrm{~cd} / \mathrm{m}^{2}$ increments. This process continued for 10 days, at which point, performance during the light $\left(\mathrm{S}^{\mathrm{D}}\right)$ and dark $\left(\mathrm{S}^{\Delta}\right)$ trials was no longer statistically different.

\section{Results}

\section{Training}

The success of discrimination responding was assessed using two dependent measures: percentage of response during $\mathrm{S}^{\mathrm{D}}$ periods and percentage of response during $\mathrm{S}^{\Delta}$ periods. Discrimination was achieved when the percentage of $\mathrm{S}^{\mathrm{D}}$ response was significantly higher than that of $\mathrm{S}^{\Delta}$.
A $2 \times(8)$ mixed model analysis of variance (ANOVA) was used to assess training with disease condition acting as the between factor and day of training as the within factor.

\section{Percentage of $\mathrm{S}^{\mathrm{D}}$ responding}

Main effect testing for disease condition on the dependent measure of $\mathrm{S}^{\mathrm{D}}$ responses was found to be nonsignificant (F $=1.182, P>0.28)$. When collapsing across the day of training variable, taiep and normal rats responded similarly during the $\mathrm{S}^{\mathrm{D}}$ periods.

As shown in Figure 1, main effect testing for the day of training was found to be significant $(\mathrm{F}=9.609, P=0.001)$, demonstrating an increase in $\mathrm{S}^{\mathrm{D}}$ response percentage over the course of training. Tests of the condition by day of training interaction were found to be nonsignificant $(\mathrm{F}=1.141$, $P=0.345)$, suggesting that no differences in response rate existed between taiep and normal rats.

\section{Percentage of $S^{\Delta}$ responding}

Main effect testing for disease condition on the dependent measure of $\mathrm{S}^{\Delta}$ responses was found to be nonsignificant (F $=0.001, P>0.98)$. When collapsing across the day of training variable, taiep and normal rats responded similarly during the $\mathrm{S}^{\Delta}$ periods.

As demonstrated in Figure 1, main effect testing for the day of training was found to be significant $(\mathrm{F}=28.128$, $P<0.001$ ), demonstrating a decrease in the $\mathrm{S}^{\Delta}$ response percentage over the course of training. Tests of the condition by day of training interaction were found to be nonsignificant $(\mathrm{F}=2.215, P>0.068)$, suggesting no differences in response rates between taiep and normal rats.

\section{Visual deficits}

For the purpose of assessing visual deficits, the dependent variable "error" was created. The error index was calculated by summing the numbers of trials without a response in the presence of $S^{\mathrm{D}}$ (false negatives) and the number of responses during the $\mathrm{S}^{\Delta}$ (false positives) divided by the total number of opportunities to respond. This value represents the proportion of errors made, with a value of 0 representing no errors and 1 representing an error on every trial.

A $2 \times(9)$ mixed-model ANOVA was used to assess the impact of disease condition on index at each of the nine levels of illumination. Post hoc analyses were completed where appropriate using Scheffe's planned comparisons. Main effects tests for disease condition were not significant $(\mathrm{F}=0.218$, $P=0.641$ ), suggesting that, when collapsing across levels 


\section{A}

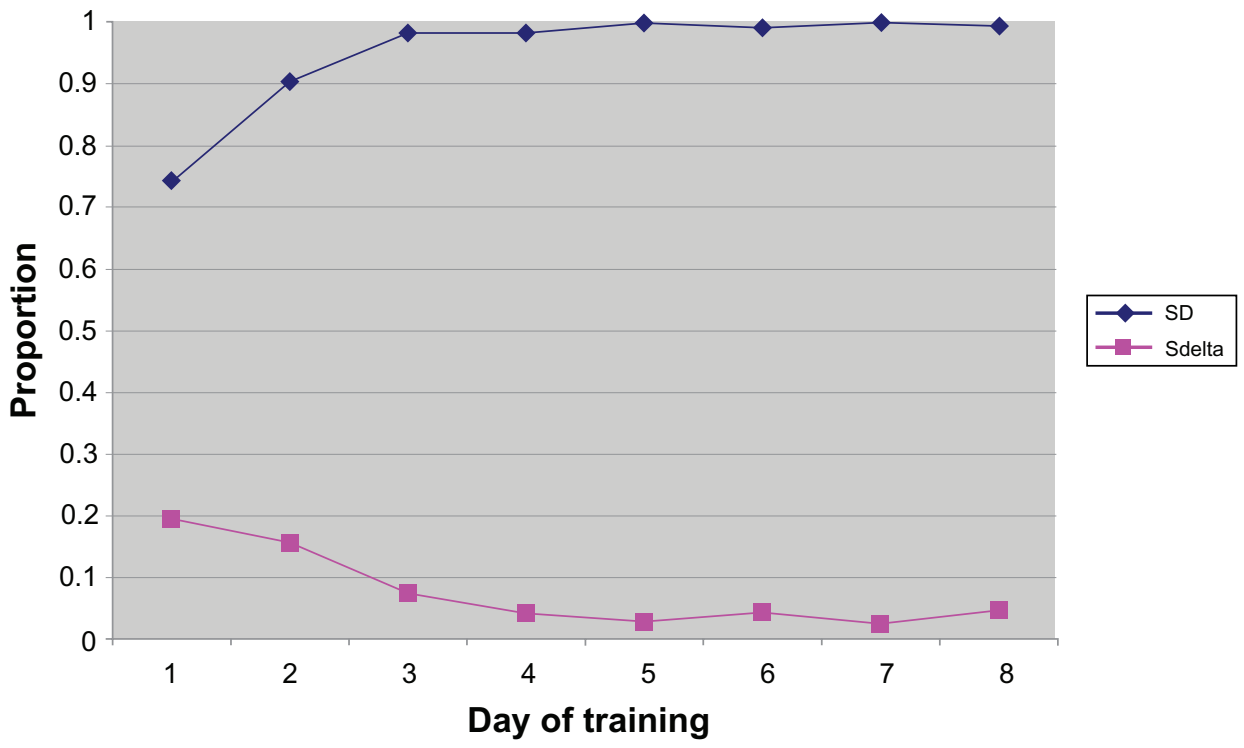

B

Average proportion-taiep

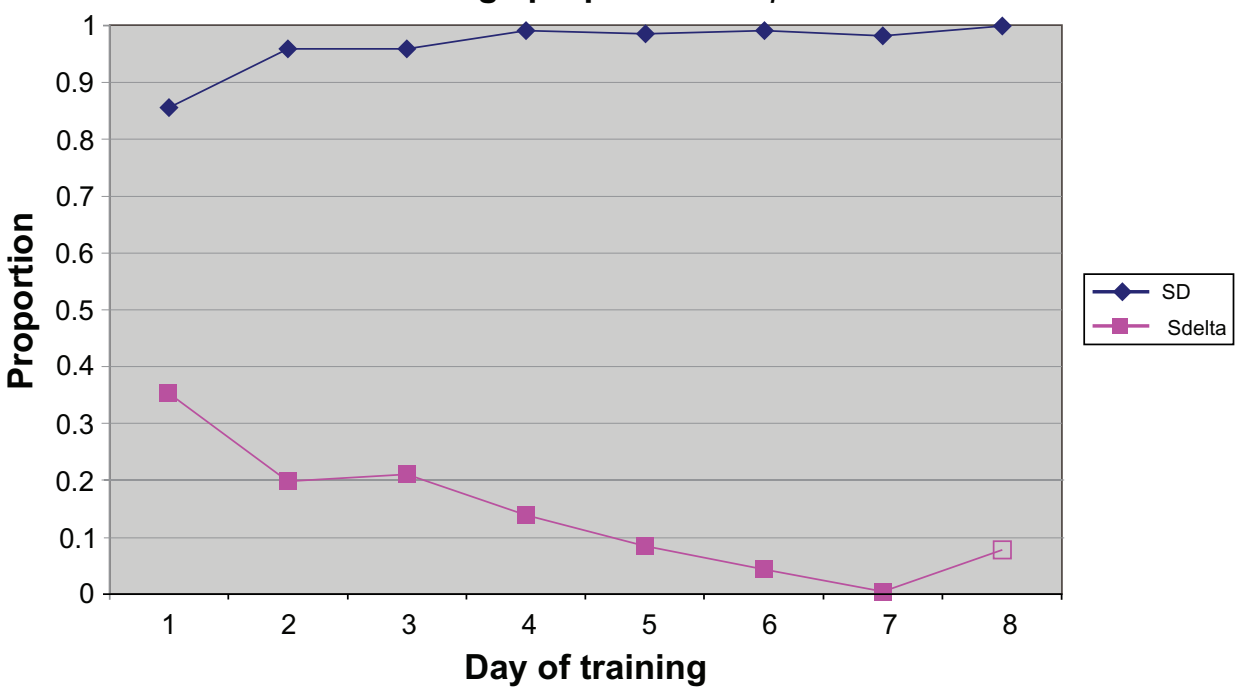

Figure I Stimulus discrimination training for both normal (A) and taiep (B) animals.

of illumination, no differences existed between normal and taiep rats.

As shown in Figure 2, main effect tests for illumination magnitude were found to be significant ( $\mathrm{F}=36.320$, $P=0.001)$, demonstrating that, when ignoring disease condition, the magnitude of illumination had a statistically significant effect on index.

The interaction between disease condition and luminance magnitude was found to be nonsignificant ( $\mathrm{F}=0.123$, $P=0.999)$, as is depicted in Figure 3. This result suggests that differences in index were solely a function of luminance magnitude and not affected by disease condition.

\section{Stimulus discrimination}

Examination of error rates for both conditions suggests that visual discrimination for both taiep and normal rats failed between -5.5 and $-6.0 \log \mathrm{cd} / \mathrm{m}^{2}$, which is consistent with expected visual thresholds. These findings do not support visual deficits in the taiep myelin mutants, suggesting instead that visual sensitivity of the diseased and normal conditions are nearly identical.

\section{Discussion}

The taiep myelin mutant's long life span makes it unique amongst animals with similar diseases of myelin, as its 


\section{A Luminance main effect-normal}

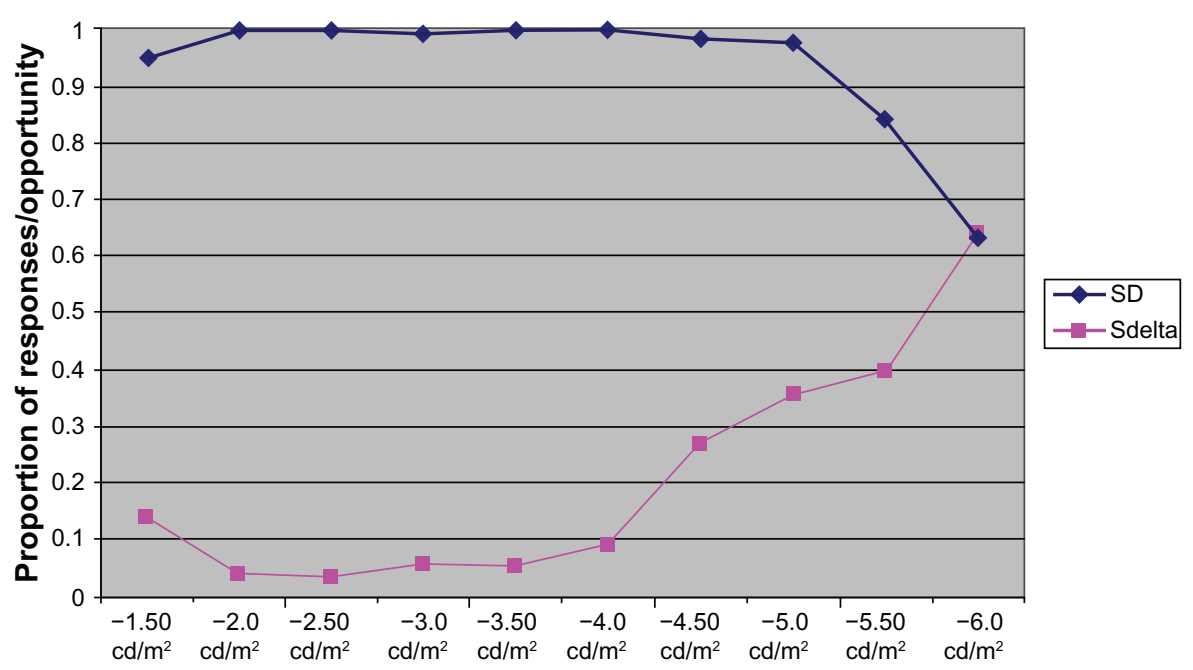

Luminance
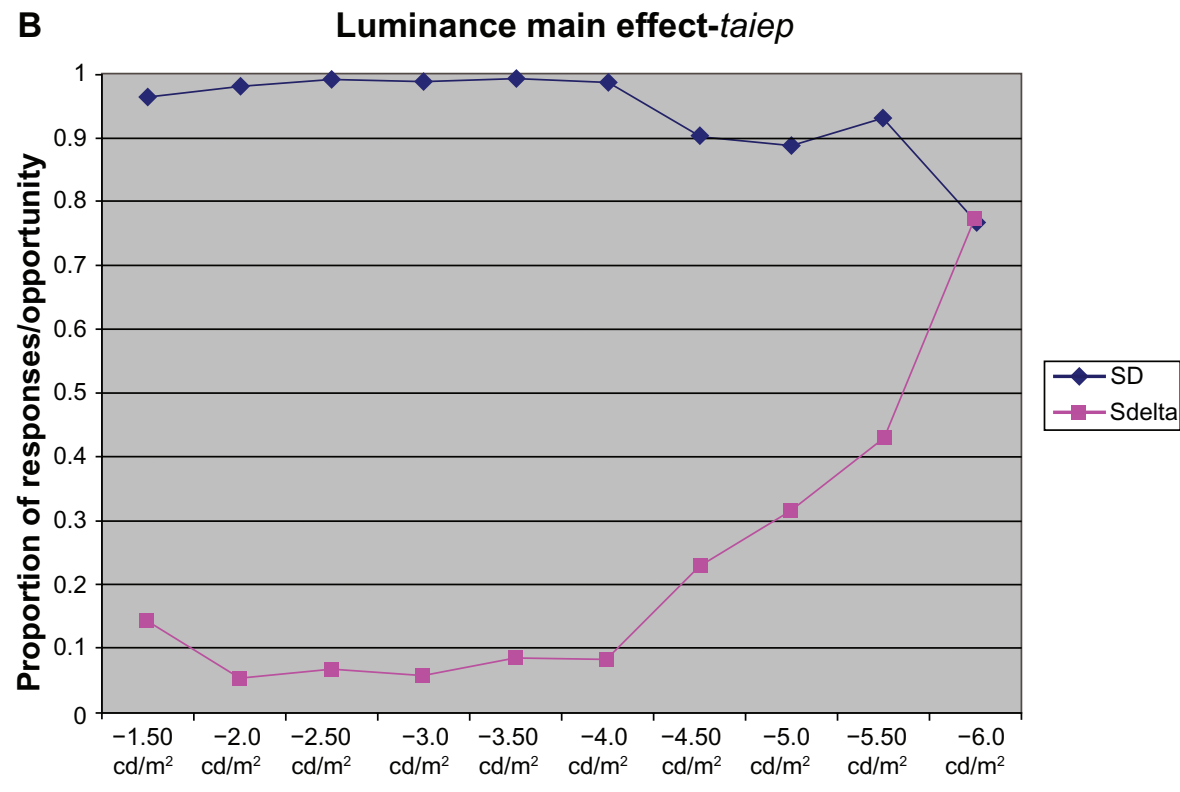

Luminance

Figure 2 Successful responding in the presence of the stimulus light as a function of magnitude for both normal (A) and taiep (B) animals. Abbreviation: cd, candela.

relative longevity makes it possible to carry out detailed investigations of the physiological, behavioral, and sensory manifestations of the disease.

The primary mechanism in the myelin aberrance of the taiep rat is linked to a malfunction in microtubule production. ${ }^{3}$ Lunn et al found that this microtubule proliferation closely mirrored the developmental progression of demyelination in the optic nerve, ventral columns, and corticospinal tracts of the taiep mutant. ${ }^{8}$ The severity of the damage to the optic nerve myelin suggests that visual deficits may be likely in the taiep mutant. Lunn et al also reported that, by 20 days of age, taiep mutants already demonstrate significantly less myelin than do similarly aged normal rats. ${ }^{8}$ The schism between normal and taiep optic nerve myelin continues throughout the progression of the disease and, by 150 days, the optic tracts of taiep rats are nearly devoid of myelin.

The operant technique used in this study provided us with an opportunity to explore the possibility of visual deficits in the taiep visual system. Acquisition of the stimulus discrimination progressed at similar rates to normal rats, and at no time were any differences between the groups on the two dependent measures statistically significant. Both groups 


\section{Disease condition $\mathrm{x}$ luminance}

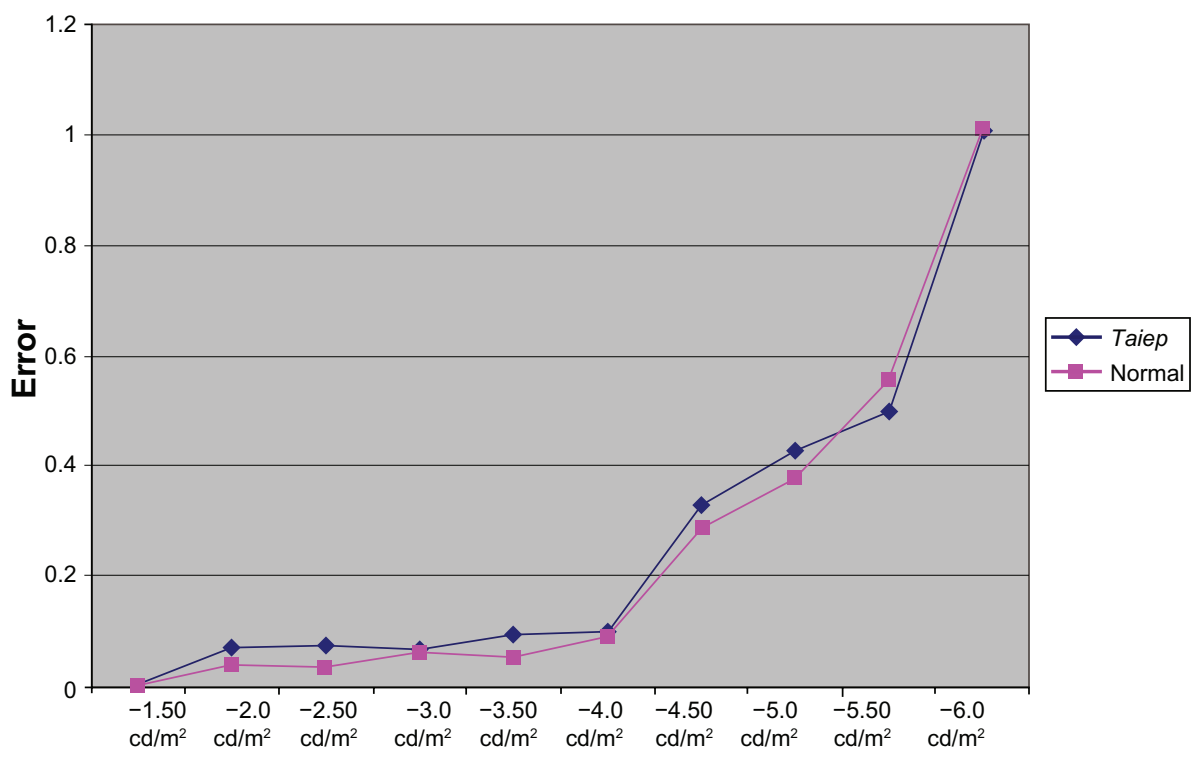

Luminance value

Figure 3 Proportion of response errors (false-negatives + false-positives/total trials) as a function of light magnitude in normal and taiep animals. Abbreviation: cd, candela.

of animals successfully recognized and responded in the presence of the stimulus near perfectly by the final day of training.

Despite the severe demyelination of the taiep optic nerve, no visual deficits were detected by this procedure. Testing of the interaction between luminance value and disease condition was not significant, suggesting that both normal and taiep animals performed similarly as the level of the light stimulus was decreased. Index values increased from 0.5 to 1.0 at luminance values $-5.5 \mathrm{~cd} / \mathrm{m}^{2}$ and $-6.0 \mathrm{~cd} /$ $\mathrm{m}^{2}$, indicating that it is between these two values that the light is no longer detectable by any of the rats. This result is consistent with VEP studies of albino and lends further support to the use of operant techniques for the investigation of visual thresholds in rodents; $;^{14}$ the consistency of these data lends further support to the use of operant techniques for the investigation of visual thresholds in rodents. Collectively, this evidence suggests that taiep rat vision does not differ in light sensitivity from normal rats. Previous work by Benitez et al demonstrates a progressive reduction in amplitude and latency in VEP in the occipital cortex of taiep rats when compared to controls. ${ }^{16}$ Our results suggest that the change in wave forms described by Benitez does not manifest in visual deficits, underscoring the role of behavioral techniques in identifying the practical manifestation of neurodegenerative disease in vivo.
It is important to note that the taiep animals were tested fairly early in the development of the disease. Though the operant technique utilized is less susceptible than other techniques to motor deficits contaminating the results, later symptoms of taiep (ataxia, paralysis, seizure) are very severe and would make successful testing under the operant paradigm difficult. To avoid this possibility, examination of the visual thresholds took place during a period in the disease in which the symptoms were at a reasonable level, characterized primarily by tremors. Lunn et al showed that, at the age investigated in this experiment, the optic nerve of taiep rats was significantly compromised, though much of the myelin was still intact and relatively healthy. ${ }^{8}$ Despite the myelin deficits at the time of testing, failure to find visual deficits may still be related to the disorder's progressive nature. It has recently been suggested that the symptoms of myelin diseases may largely be a function of axonal degeneration. ${ }^{17}$ Oligodendrocytes, which serve to myelinate axons, may also play an axonotrophic role via other biochemical mechanisms. Wilkins et al report that, at the time of testing (between 2-3 months of age), taiep animals display a loss of only $25 \%$ of optic nerve axons, demonstrating a slower progression in axonal degeneration when compared to the loss of myelin. ${ }^{17}$ It may be that visual deficits are better predicted as a result of axonal degeneration rather than demyelination. ${ }^{1,2,16}$ Future work utilizing the 
technique described may allow for the tracking of visual deficits throughout the progression of the disease and provide evidence that deficits follow a time course more closely tied to axonal degeneration than to the demyelination itself.

\section{Disclosure}

The authors report no conflicts of interest in this work.

\section{References}

1. McDonald WI, Sears TA. The effects of experimental demyelination on conduction in the central nervous system. Brain. 1970;93(3):583-598.

2. McDonald WI, Sears TA. Focal experimental demyelination in the central nervous system. Brain. 1970;93(3):575-582.

3. Duncan ID, Lunn KF, Holmgren B, Urba-Holmgren R, Brignolo-Holmes L. The taiep rat: A myelin mutant with an associated oligodendrocyte microtubular defect. J Neurocytol. 1992;21(1):870-884.

4. Anch AM, Powell E, Bloom C, Dyche J, Faulkner K, Richter RR. Locomotor analysis of the taiep rat. J Gen Psychol. 2000;127(4):412-425.

5. Li FY, Song J, Duncan ID. Mapping of taiep rat phenotype to rat Chromosome 9. Mamm Genome. 2003;14(10):703-705.

6. Leon Chavez BA, Guevara J, Galindo S, et al. Regional and temporal progression of reactive astrocytosis in the brain of the myelin mutant taiep rat. Brain Res. 2001;900(1):152-155.

7. Flores G, Flores J, Mena R, Valencia J. Mutant taiep rats exhibit an increase in D1 binding in basal ganglia. Brain Res. 2002;956(1):24-29.
8. Lunn K, Baas P, Duncan ID. Microtubule organization and stability in the oligodendrocyte. J Neurosci. 1997;17(1):4921-4932.

9. Powell E, Anch AM, Dyche J, Bloom C, Richter RR. The splay angle: a new measure of assessing neuromuscular dysfunction in rats. Physiol Behav. 1999;76(5):819-821.

10. Black JA, Dib-Hajj S, Baker D, Newcombe J, Cuzner ML, Waxman SG. Sensory neuron-specific sodium channel SNS is abnormally expressed in the brains of mice with experimental allergic encephalomyelitis and humans with multiple sclerosis. Proc Natl Acad Sci U S A. 2000; 97(21):11598-11602.

11. Holmgren B, Urbá-Holmgren R, Riboni L, Vega-SaenzdeMiera EC. Sprague Dawley rat mutant with tremor, ataxia, tonic immobility episodes, epilepsy and paralysis. Lab Anim Sci. 1989;39(1):226-228.

12. Muñoz Tedó C, Herreros de Tejada P, Green DG. Behavioral estimates of absolute threshold in rat. Vis Neurosci. 1994;11(6):1077-1082.

13. Blough D, Blough P. Animal psychophysics. In: Handbook of Operant Behavior. New York, NY: Academic Press; 1970.

14. Prusky GT, West PW, Douglas RM. Behavioral assessment of visual acuity in mice and rats. Vision Res. 2000;40(16):2201-2209.

15. Accornero N, De Vito G, Rotunno A, Perugino U, Manfredi M. Critical fusion frequency in MS during mild induced hyperthermia. Acta Neurol Scand. 1989;79(6):510-514.

16. Benitez J, Holmgren B, Eguibar J, Roncagliolo M. Multimodal sensory evoked potentials in a rat model of demyelinating diseases. Electroen Clin Neuro. 2007;103(1):189-189(1).

17. Wilkins A, Kondo Y, Song J, et al. Slowly progressive axonal degeneration in a rat model of chronic, nonimmune-mediated demyelination. J Neuropathol Exp Neurol. 2010; 69(12):1256-1269.
Degenerative Neurological and Neuromuscular Disease

\section{Publish your work in this journal}

Degenerative Neurological and Neuromuscular Disease is an international, peer-reviewed, open access journal focusing on research into degenerative neurological and neuromuscular disease, identification of therapeutic targets and the optimal use of preventative and integrated treatment interventions to achieve improved outcomes, enhanced

\section{Dovepress}

survival and quality of life for the patient. The manuscript management system is completely online and includes a very quick and fair peer-review system. Visit http://www.dovepress.com/testimonials.php to read real quotes from published authors. 